
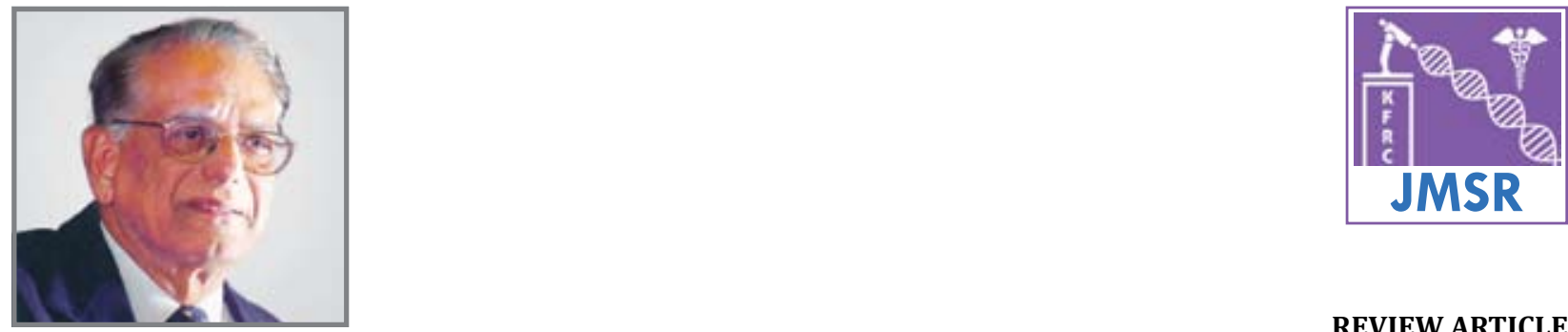

Prof. Kakarla Subbarao

REVIEW ARTICLE

\title{
Tumoral lesions of bone
}

\author{
Prof. Kakarla Subbarao ${ }^{1, *}$ \\ ${ }^{1}$ Chairman, KIMS Foundation and Research Centre, Minister Road, Secunderabad - 500003, Telangana, India
}

*Corresponding author: Prof. KakarlaSubbarao, MS, D.Sc. (HON), FRCR, FACR, FICP, FSASMA, FCCP, FICR, FCGP, Chairman, KIMS Foundation and Research Centre, Minister Road, Secunderabad 500003, Telangana, India. Email: subbaraokakarla25@gmail.com

Received 10 May 2014; Accepted 14 June 2014

Citation: Kakarla Subbarao (2014) Tumoral lesions of bone. J Med Sci Res 2(3):161-168. DOI: http://dx.doi.org/10.17727/ JMSR.2014/2-030

Copyright: (c) 2014 KIMS Foundation and Research Centre. All Rights Reserved.

\section{Introduction}

In daily radiological practice, one often encounters problems in differentiating real bone tumors from tumoral bone lesions. Bone reacts in limited ways for any stimulus of developmental or acquired nature. These pathological reactions are reflected in radiology and imaging investigations. These include new or reactive bone which is dense, lysis of the bone are non-mineralized matrix which is lucent and periosteal reaction in several patterns. It may be a combination of these lesions. Conventional radiology is often adequate to diagnose tumoral lesions of bone, without further imaging studies. Tumoral lesions of bone are of several types which are mentioned in table 1 . These lesions pose a diagnostic problem but can be differentiated from tumors by detailed study. This review attempts at solving these problems by studying the radiological findings in each entity. However, many of these radiological findings should be evaluated in the light of clinical setting where age is an important factor. The common tumoral lesions are listed in table 1.
Table 1: The common tumoral lesions.

Bone islands
Fibro osseous lesions - Ossifying fibroma, osteofibrous
dysplasia, fibrous dysplasia
Fibrocortical defects
Non-ossifying fibroma
Simple bone cyst (SBC)
Aneurysmal bone cyst (ABC)
Post traumatic bone lesion
Dystrophic calcification / Ossification
Granulomas - Histiocytosis X \& sarcoid
Infective lesions - Bacterial, fungal
Pseudotumor hemophili
Pseudotumor gout
Brown tumor
Bone infarct
Intraosseous ganglion

\section{Bone islands}

These are often encountered as circumscribed homogenous dense lesions on plain films. The size may range from few millimetres to a centimetre and may simulate osteoid osteoma which has typical radiological findings such as a nidus and surrounding reaction (Figure $1 \mathrm{ab}$ ). It may grow and assume a large size when it is called a giant bone island (Figure 1c). Developmentally, these bone islands are cortical inclusions in trabecular bone. They may be multiple and involve many bones, and should not be mistaken for osteopoikilosis (Figure 1d). 


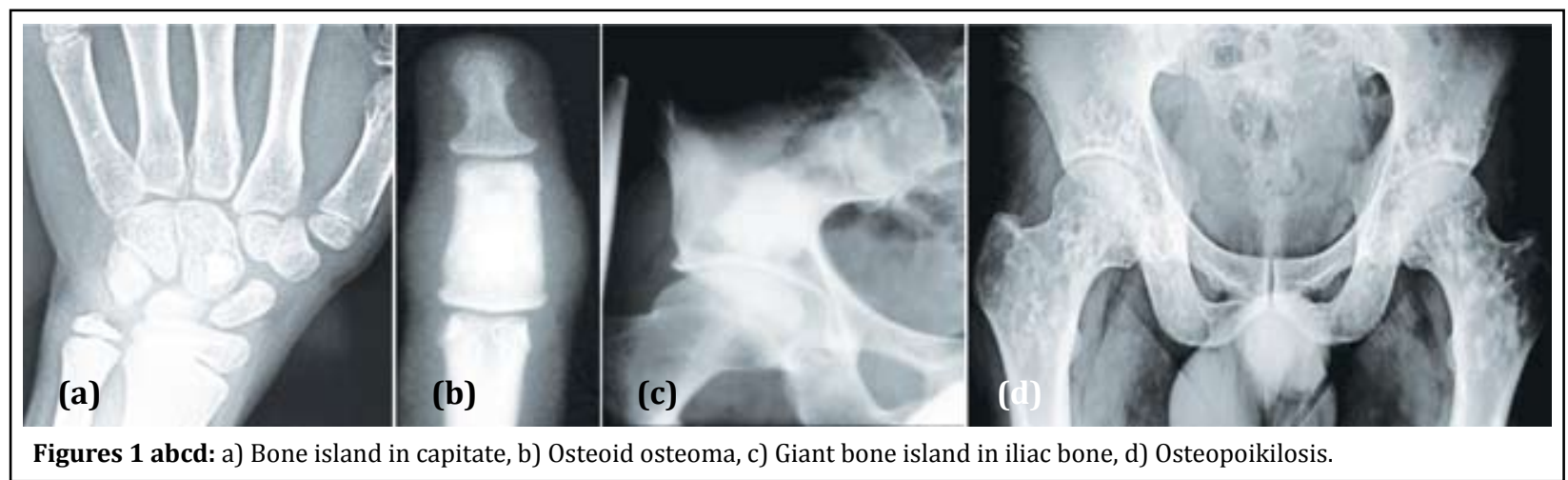

\section{Fibro osseous lesions}

\section{Ossifying fibroma}

It may be encountered in childhood, from infancy to 10 years of age. Radiologically, it is a lytic lesion with multiloculated sclerotic borders. Anterior bowing of the tibia is a common feature (Figure 2ab). Spontaneous regression is well known and is often called a "leave me alone lesion". However, subsequent to trauma or surgery, pseudo arthrosis may develop. It may also occur in the bones of the jaw.

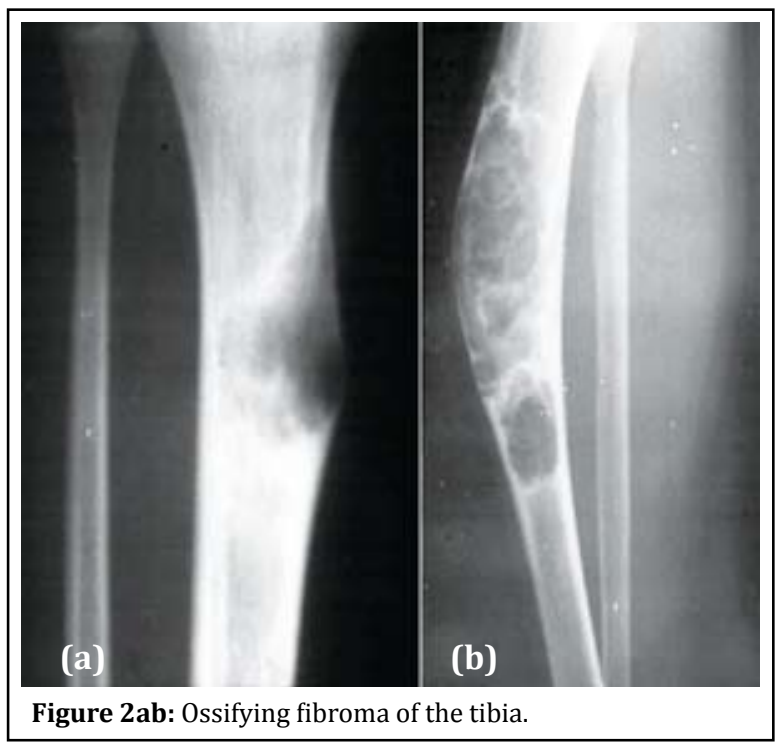

\begin{tabular}{|c|c|c|}
\hline & Ossifying fibroma & Fibrous dysplasia \\
\hline $\begin{array}{l}\text { Age \& } \\
\text { radiological } \\
\text { features }\end{array}$ & $\begin{array}{l}0 \text {-10 yrs } \\
\text { lytic expanding } \\
\text { Intramedullary } \\
\text { lesion with cortical } \\
\text { involvement }\end{array}$ & $\begin{array}{l}\text { Any age } \\
\text { lytic expanding } \\
\text { lesion with ground } \\
\text { glass matrix }\end{array}$ \\
\hline Site & Mid shaft & Any part of the bone \\
\hline Complications & $\begin{array}{l}\text { Pseudo arthrosis } \\
\text { Spontaneous } \\
\text { regression }\end{array}$ & $\begin{array}{l}\text { Rare } \\
\text { Not known }\end{array}$ \\
\hline
\end{tabular}

Some authors consider ossifying fibroma and osteofibrous dysplasia as a spectrum of the same entity.

\section{Osteofibrous dysplasia}

It is also named as Kempson-campanacci lesion. It commonly involves tibia shaft. Radiologically, an elongated lytic lesion with areas of sclerosis and anterior bowing (Figure 3abc).

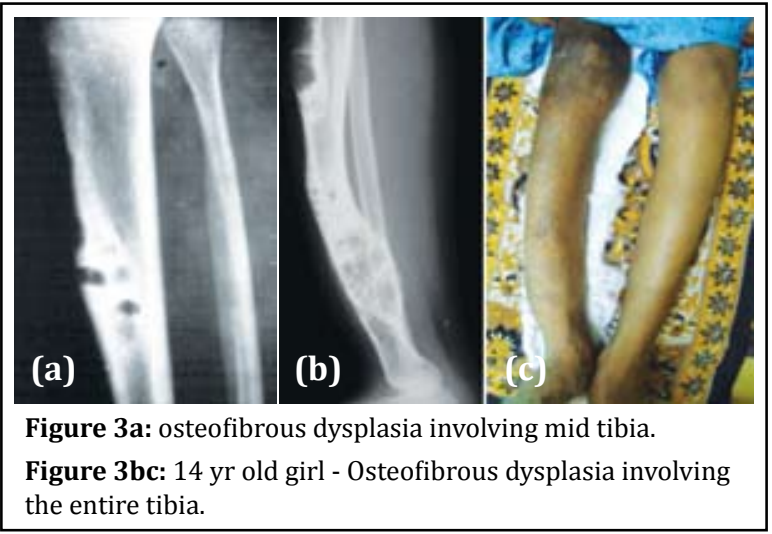

\section{Fibrous dysplasia}

Most common bone dysplasia where fibrous aberration occurs replacing the bone as a developmental error. A defective gene exists in the cells that form bone and other tissues. It may be monostotic and may simulate a benign or malignant bone tumor. However, the radiological features such as transitional zone in an expanding lytic area, thick sclerotic rind, ground glass appearance of the matrix and local deformity of bone differentiate from benign bone tumors (Figure 4abcd). On rare occasions chondroid type of calcifications may be seen. Pathological fractures may occur and the lesion may be aggressive simulating malignancy.

\section{Fibrous cortical defects}

Fibrous cortical defects are developmental errors 
where periosteal membrane is included in the cortex of the long bones (Figure 5). It is encountered in children and spontaneous resolution is known. On the other hand, they may progress to non-ossifying fibroma.

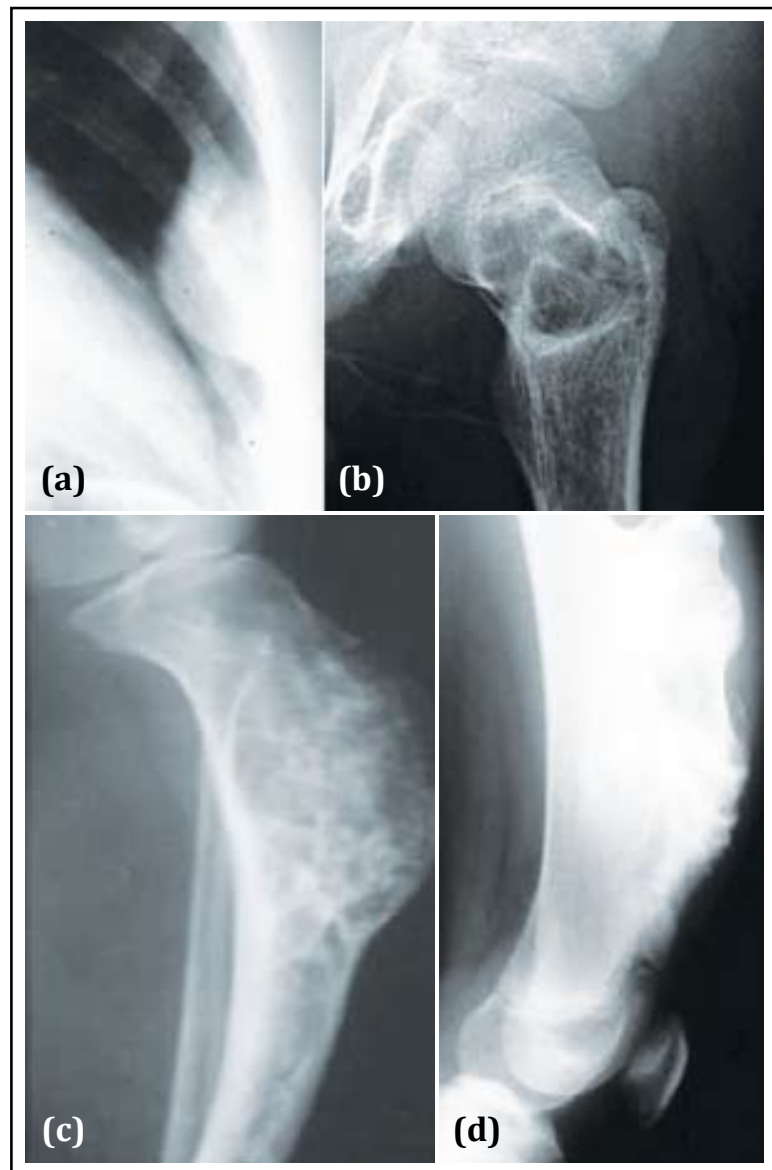

Figure 4abc: a) Fibrous dysplasia of rib, b) neck of femur with pathological fracture, c) aggressive lesion simulating cartilagenous lesion.

Figure 4d: Sessile exostosis simulating fibrous dysplasia.

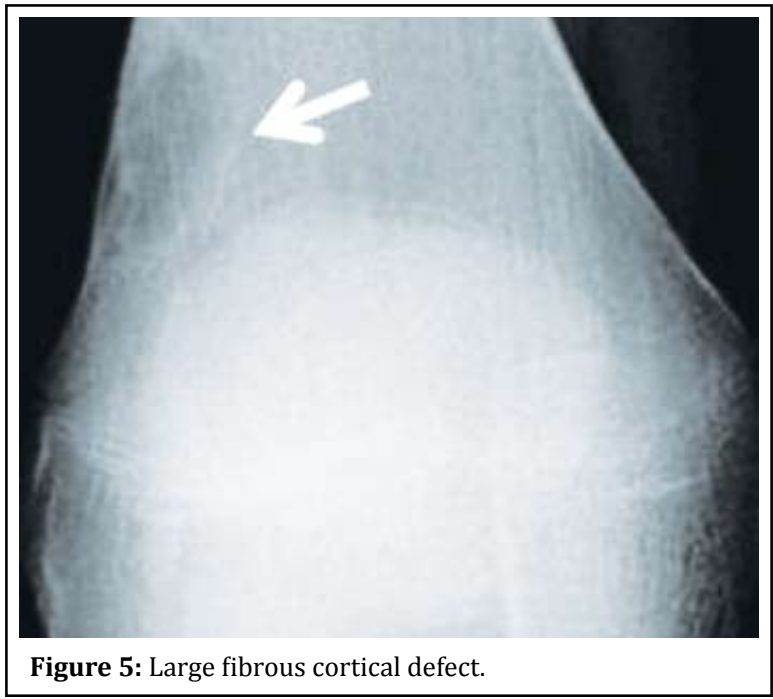

\section{Non ossifying fibroma}

These are cortical oriented fibrous lesions often encountered between 12 and 30 years. Lower limb bones are most commonly involved. Radiologically, a large, oblong lytic lesion with sclerotic borders (Figure 6ab). The patient clinically presents with a pathological fracture. In the healing phase, new bone forms from the periphery (Figure 6c).

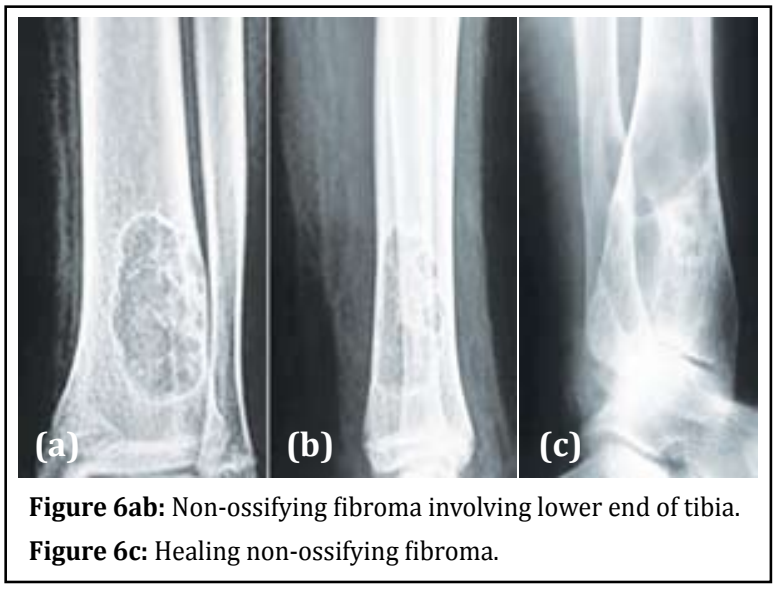

\section{Simple bone cyst (SBC)}

It is also named as unilocular bone cyst encountered in children and young adults in long bones. Radiologically, it is vertically expanding lytic lesion with thin transitional zone in the metaphyseal area which may extend to diaphysis. Several septae may be seen and pathological fracture is not uncommon. A fallen fragment sign is classical for radiological diagnosis of simple bone cyst. This is well demonstrated in erect and supine or horizontal beam films (Figure 7ab). A simple bone cyst is occasionally seen in adults particularly in the calcaneum, iliac and other bones (Figure 7cd). This may simulate lytic metastasis (Figure 7e). Brodie's abscess/ tubercular abscess may also simulate (Figure $7 f$ ).

\section{Aneurysmal bone cyst (ABC)}

It may be primary or secondary. Secondary $A B C$ is associated with giant cell tumor, chondroblastoma, chondromyxoid fibroma, fibrous dysplasia, osteoblastoma, ewings tumor and telangiectatic osteosarcoma.

Primary $\mathrm{ABC}$ is radiologically an expanding lucent lesion with thin transitional zone located in diametaphyseal or diaphyseal area. It is encountered generally in young age but it also may be seen in adults. Pathological fractures may occur. A thin shell 


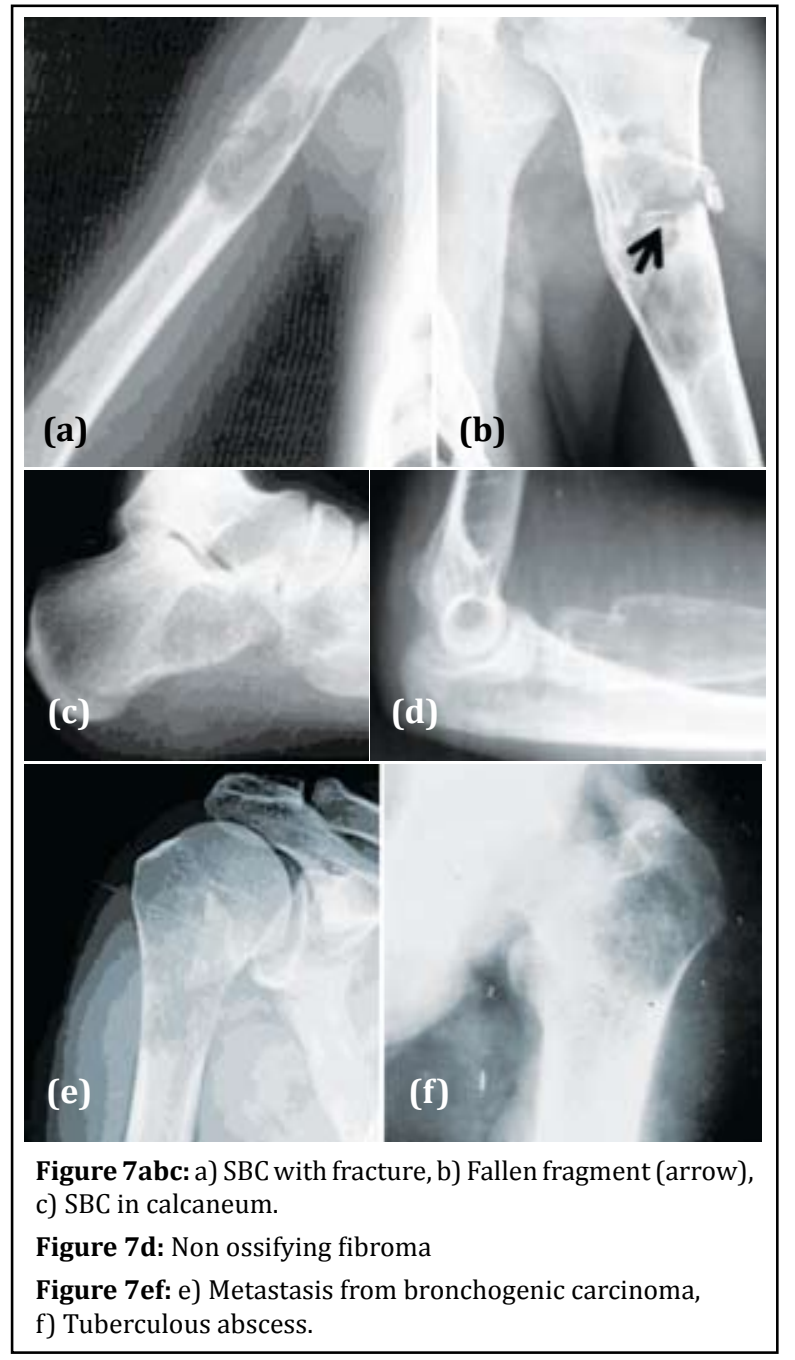

of bone is often noted (Figure 8abc). In spine, it may involve the body/pedicle/spinus process of vertebra (Figure 8d). In CT/MRI fluid fluid levels may be noted (Figure 8e). However, fluid fluid levels may also be seen in other lesions such as giant cell tumor. A solid variety of $\mathrm{ABC}$ is also seen and pathologists consider this as a variant of giant cell reparative granuloma which often occurs in the bones of hand and face.

\section{Post traumatic bone lesion}

In avulsive lesions of apophysis, the healing phase of the lesion simulates a bone tumor such as pariosteal osteosarcoma (Figure 9ab). This is due to abundant callus and associated calcified hematoma with myositis ossificans. These lesions are well circumscribed without any soft tissue swelling. If one is not familiar with this entity, these may be mistaken for benign or malignant osteogenic tumors. Post traumatic myositis ossificans can occasionally

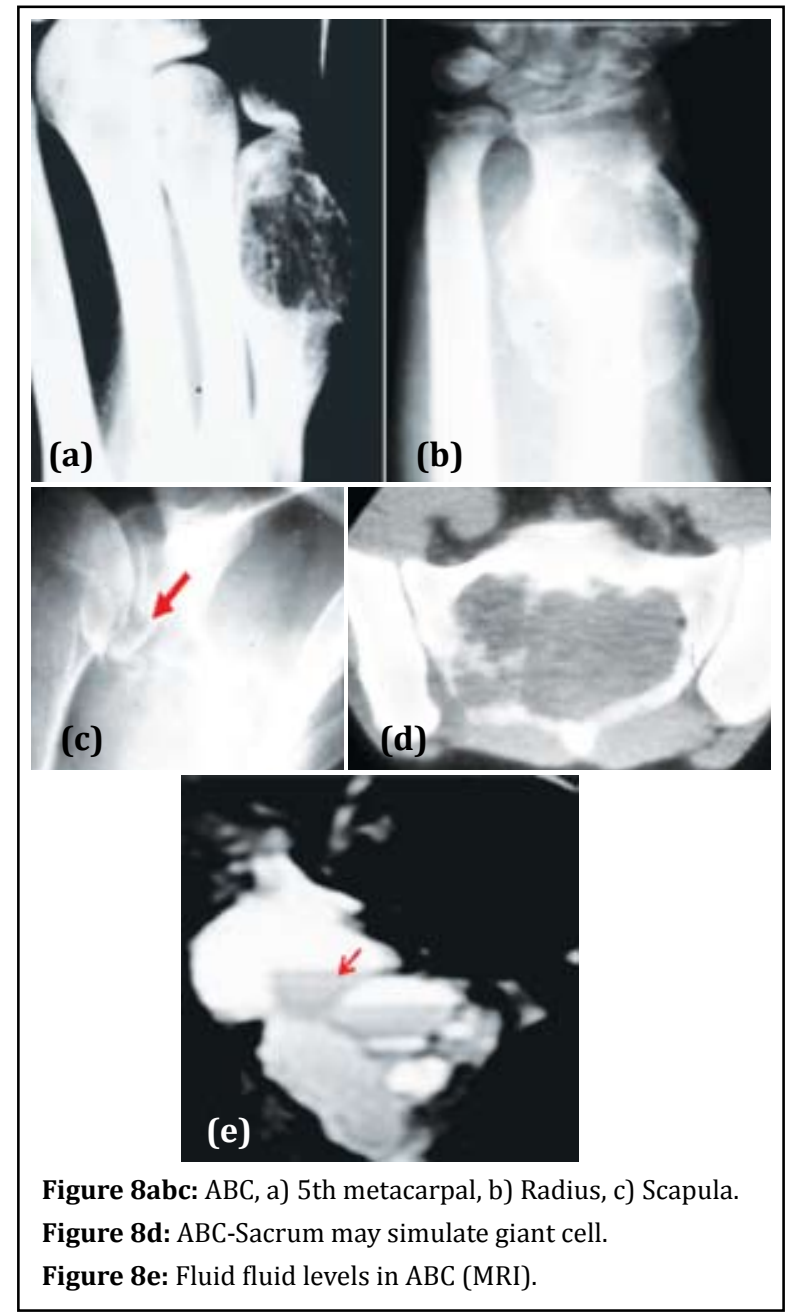

simulate osteochondroma or other benign bone lesion (Figure 9cde). In a bedridden patient due to neurological cause's dystrophic calcification/ ossification is commonly encountered in the soft tissues particularly in pelvic region which may be mistaken for an osseous tumor such as pariosteal osteosarcoma (Figure 9f).

\section{Dystrophic calcification/ ossification}

It occurs in patients with spinal cord injury or other neurological disorders. Excessive granulation tissue leads to fibroblastic proliferation leading to osteocytic activity (Figure 10ab).

\section{Granulomas}

Langerhan's histiocytosis $\mathrm{X}$ is commonly encoutered in children. However, a solitary lesion eg., eosinophilic granuloma may also be seen in adults. Radiologically, eosinophilic granuloma is a solitary lytic lesion in the medulla of a long bone with endosteal scalloping. 

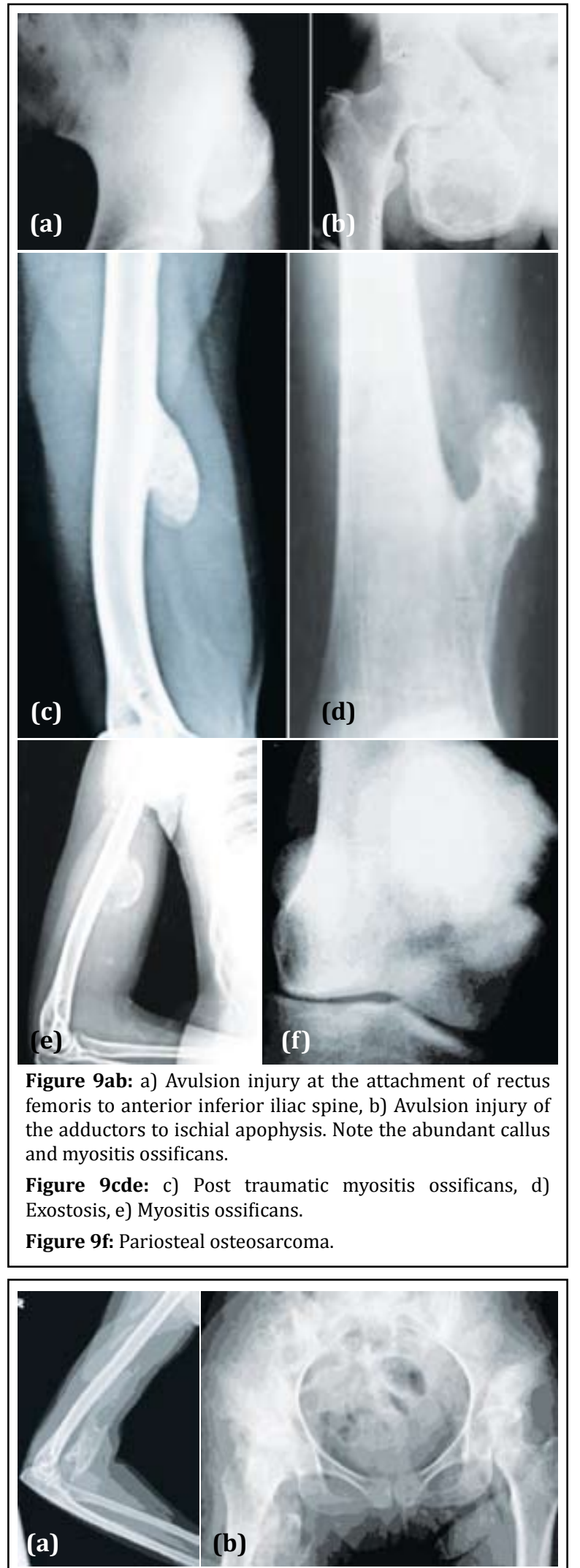

Figure 10a: Dystrophic ossification in bedridden patient.

Figure 10b: Soft tissue ossifications in a patient with paraplegia.
It may break the cortex and produce periosteal reaction (Figure 11a). When lytic it may simulate metastasis (Figure 11b). In the skull, the edges of the lytic lesion may have a bevelled edges (Figure 11c). In Hand Shuller Christian type of histiocytosis which is encoutered in young adults associated with enlargement of liver and spleen, bone lesions are multiple and may simulate lymphoma or metastasis (Figure 11d).

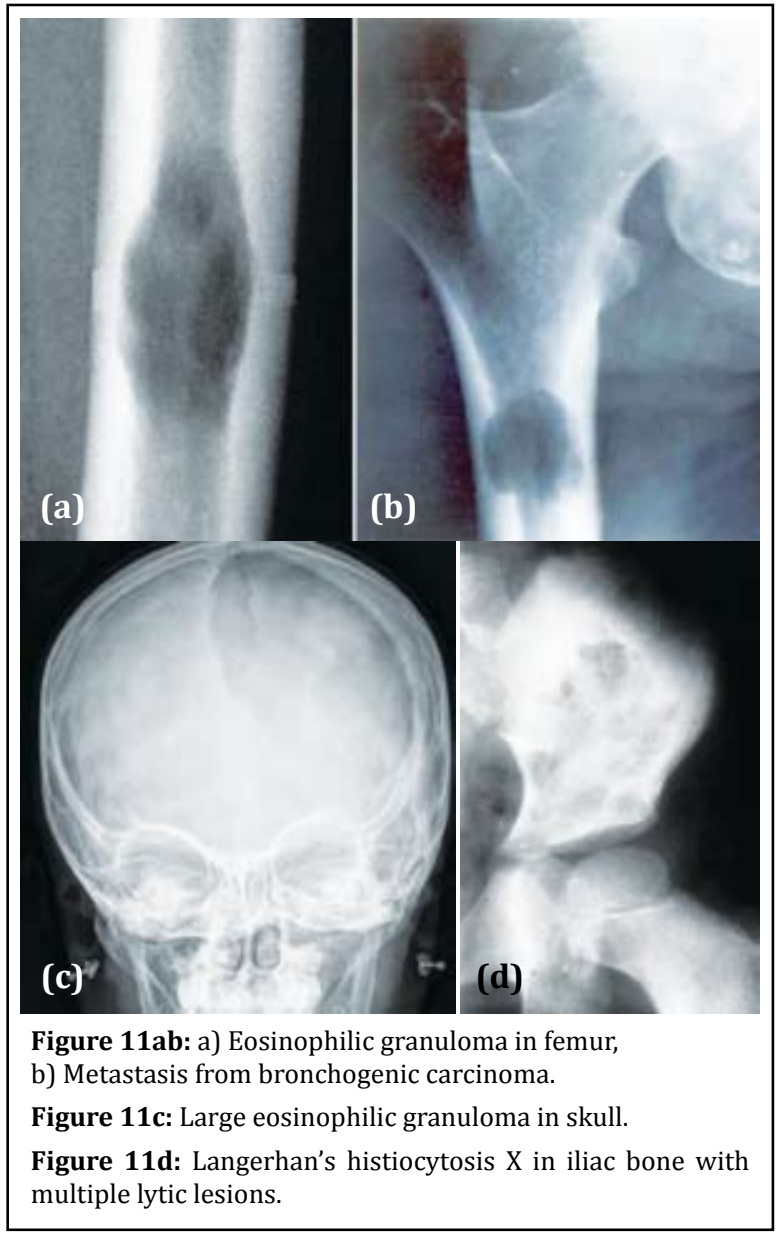

\section{Sarcoid}

Sarcoidosis involving the bones is quite rare. However, lytic lesions in the bones of hands are more common than sclerotic lesions in the skeleton.

\section{Infective lesions - Bacterial, fungal}

Inspite of introduction of several antibiotics, osteomyelitis, specially of long bones is still encountered. Brodie's abscess a form of subacute bacterial osteomyelitis may resemble a neoplasm. Radiological characterists include an oval or elongated lucent lesion in the metaphysis with sclerotic border. A small sequestrum may be 
occasionally noted along with minimal periosteal reaction (Figure 12abc).

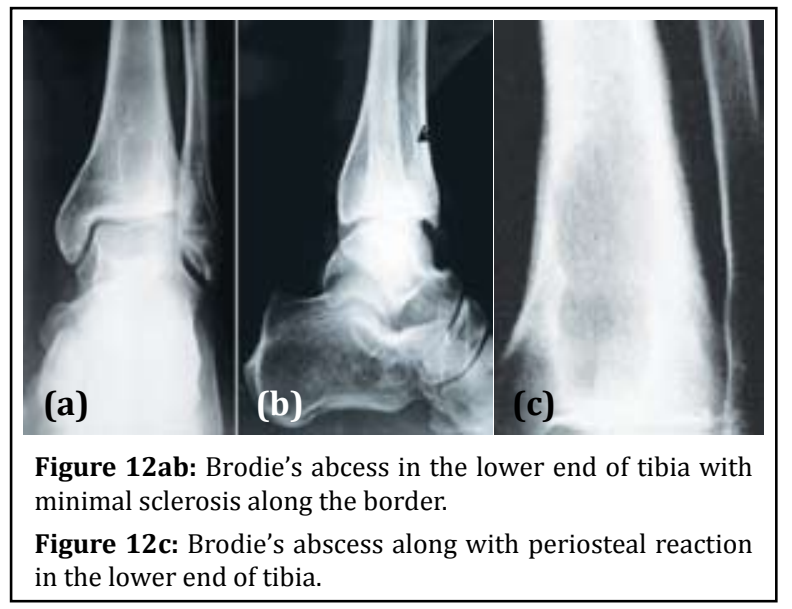

\section{Tuberculosis}

Tuberculosis (TB) of the bones is less common than joint tubeculosis. Cystic type of tuberculosis may be confused for a bone tumor. Radioloigcal characteristics are almost the same as Brodie's abscess. However, reactive new bone and periosteal reaction is minimal (Figure 13ab). Clinical and laboratory details will establish the diagnosis.

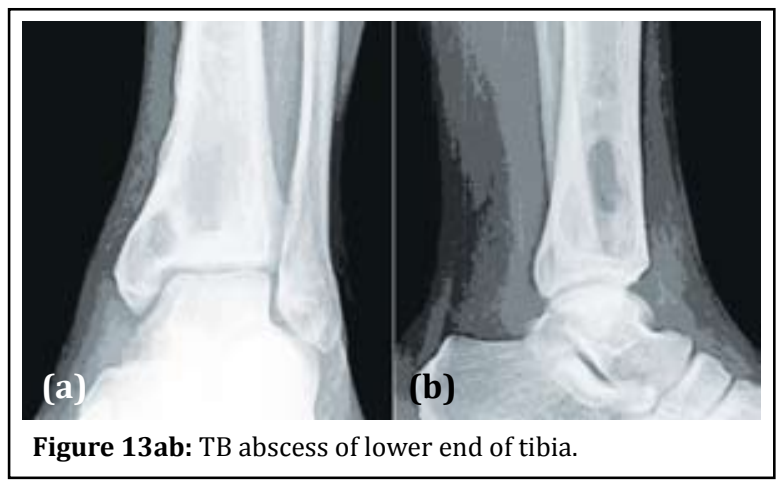

\section{Fungal infections}

Infections from nocardiosis, madura mycosis and aspergillosis rarely resemble osteoid osteoma and other tumors. These are chronic and indolent and produce various type of periosteal reaction and reactive sclerosis (Figure 14ab). When a lucent lesion with reactive sclerosis is noted, it may simulate osteoid osteoma (Figure 14c).

\section{Pseudotumor hemophili}

Hemophilic patients at times present a cystic lesion filled with blood as a rare complication. Repeated intraosseous bleeding produces a well defined uni

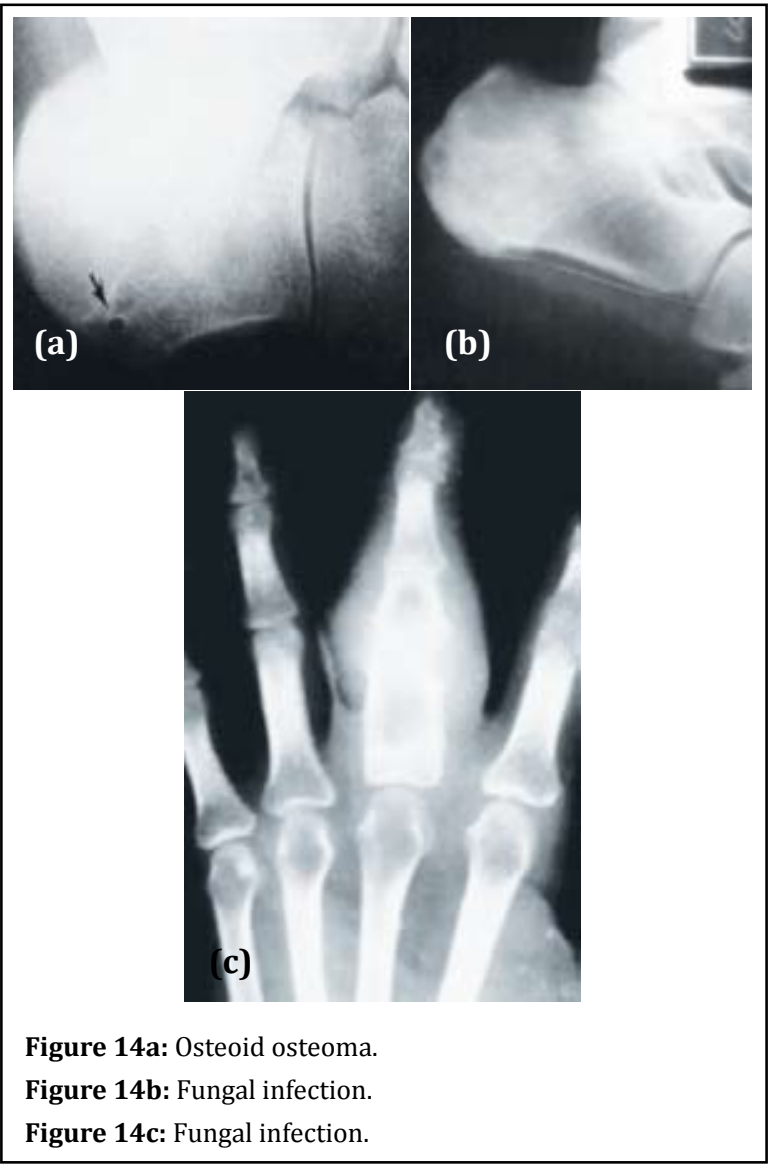

or multilocular lytic expanding lesion. These are located either in metaphysis or epiphysis (Figure 15).

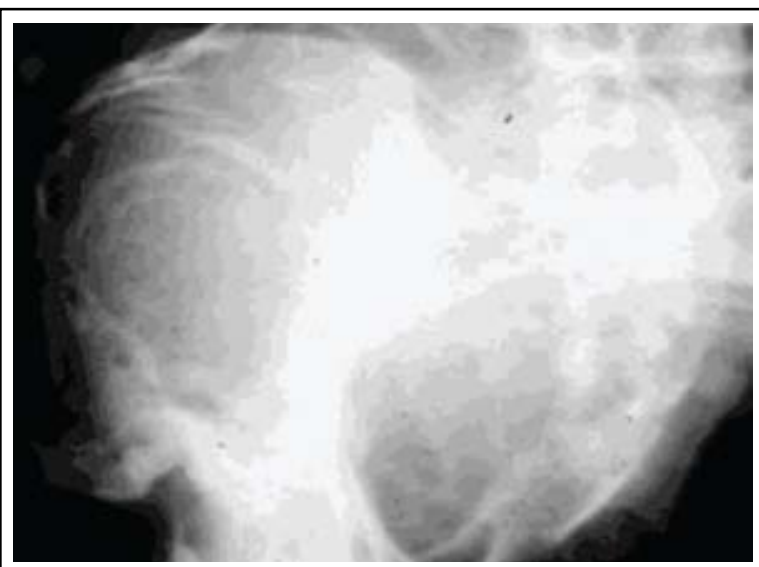

Figure 15: Pseudotumor hemophili with large lucent multilayered lesion in the iliac bone.

\section{Pseudotumor gout}

Intraosseous gouty lesions in the foot at times may simulate a tumor. However, adjacent erosions and tophi render a clue to diagnosis (Figure 16). 


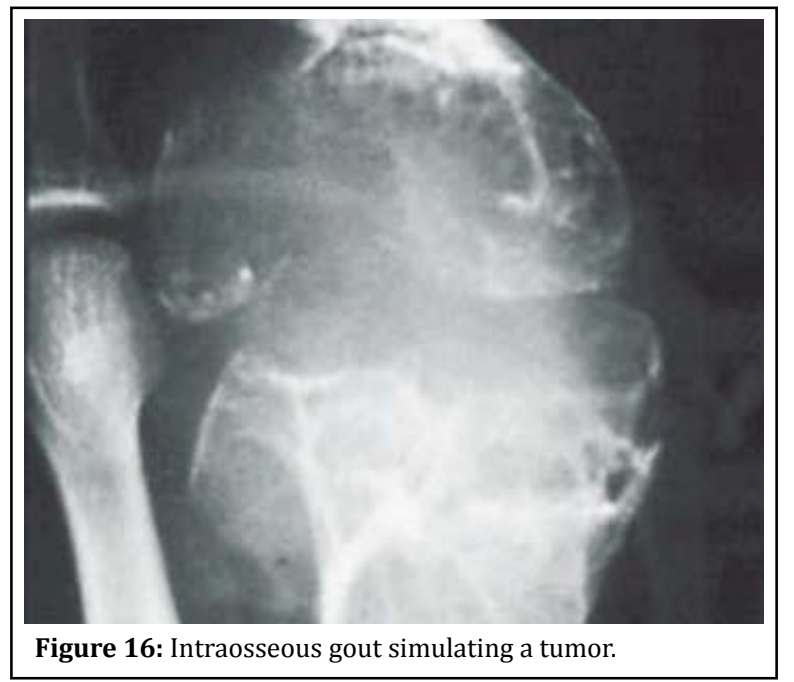

\section{Brown tumor}

Brown tumors (BT) are areas hemorrhage with fibroblasts and osteoclasts encoutered in hyperparathyroidism both in primary and secondary. These are cortically oriented osteolytic lesions occuring anywhere in the skeleton but common in long and flat bones (Figure 17a). Histiologically, resemble osteoclastoma (Figure 17b).

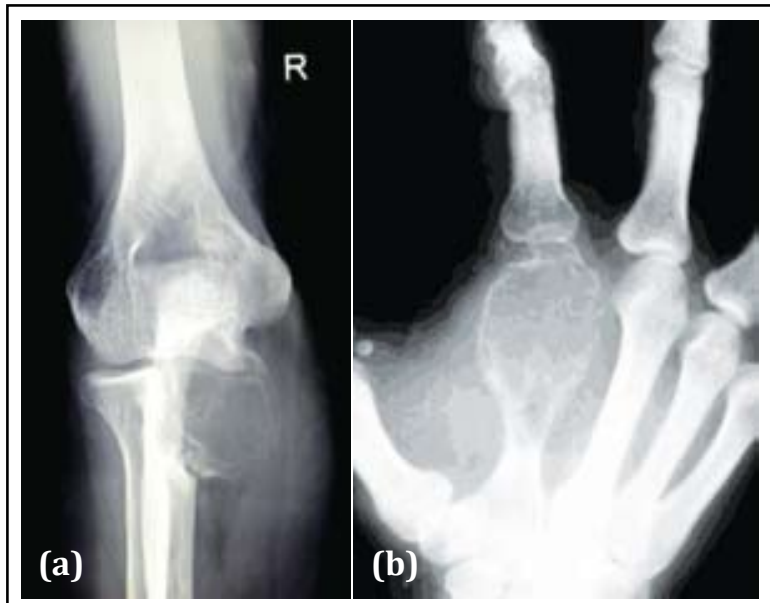

Figure 17a: Brown tumor in ulna.

Figure 17b: Giant cell tumor.

\section{Bone infarcts}

Old healed infarcts in the metaphyseal region of long bones simulate enchondroma. However, the radiological findings of an elongated lesion with serpentine calcifications and the peripherial fibroosseious membrane differentiates it from enchondroma (Figure 18abc). Occasionally the appearance may be similar to osteosarcoma (Figure 18d).

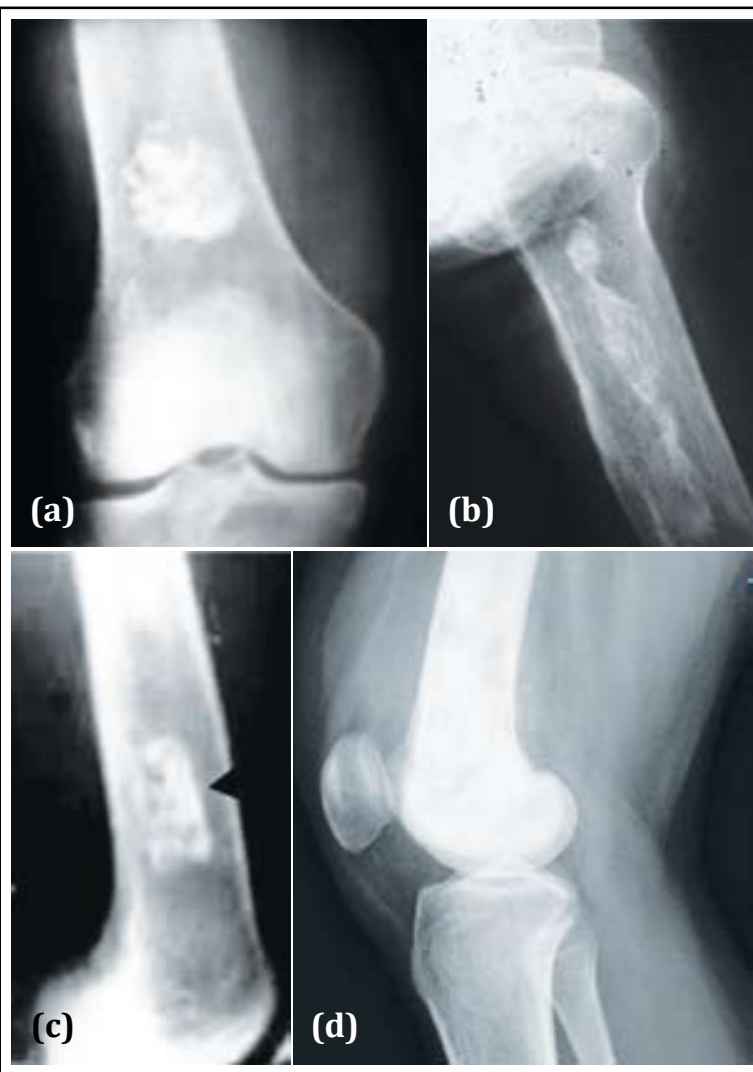

Figure 18a: Enchondroma.

Figure 18bc: b) Calcified infarct,

c) Calcified infarct in lateral view.

Figure 18d: Osteosarcoma simulating infarct.

\section{Intraosseous ganglion}

It is a subarticular lytic lesion containing synovial fluid and is often associated with degenerative joint disease. It is encountered in the proximal and distal ends of tibia. Medial malleolus is common site (Figure 19).

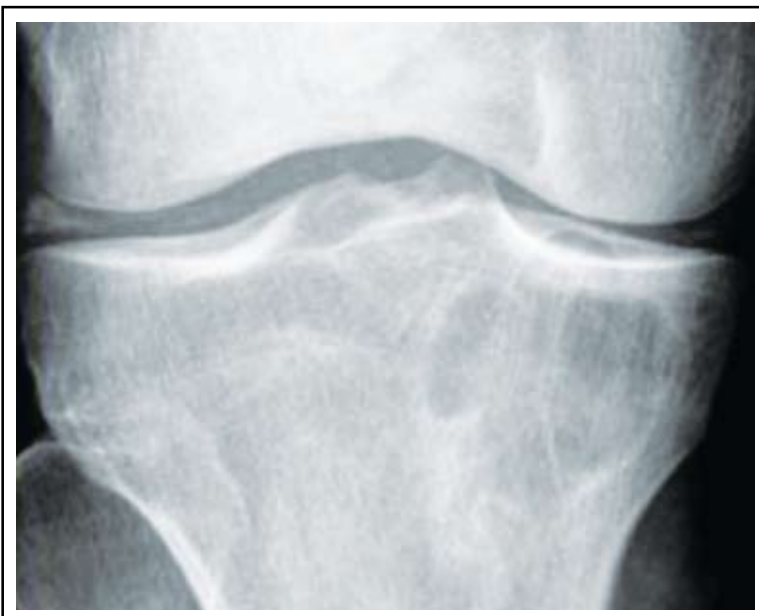

Figure 19: Intraosseous ganglion in the medial condyle of tibia. 


\section{Conclusion}

Bone reacts in a limited way and radioloigcally produces a sclerotic, lytic and a combination of these with variable periosteal reactions. Many sclerotic and lytic lesions encountered in the long bones in daily practice pose a problem differentiating benign bone tumors from tumoral lesions. These lesions should be carefully evaluated with further imaging correlating with clinical details. Occasionally, when they are aggressive may simulate primary malignant neoplasms. The varieties of tumoral lesions mimicing neoplasms are illustrated.

\section{Acknowledgements}

Acknowledgements are due to Department of Radiology and Imaging, NIMS and KIMS, Secunderabad, Telangana, India.

\section{Conflict of interest}

The author declares no conflict of interest.

\section{References}

1. Helms CA. Benign Cystic Bone Lesions. In: Brant WE, Helms CA. Fundamentals of Diagnostic Radiology. 3rd Ed. Philadelphia, PA: Lippincott Williams \& Wilkins; 2007; 1063-1085.

2. Miller TT. Bone tumors and tumor-like conditions: Analysis with conventional radiography. Radiology 2008; 246:662674.

3. Resnick D. Tumors and Tumor-Like Lesions of Bone: Radiographic Principles. In: Resnick D. Bone and Joint Imaging 2nd Ed. Philadelphia, PA: WB Saunders 1996; 979990.

4. Mhuircheartaigh JN, Yu-Ching L, Jim SW. Bone tumor mimickers: A pictorial essay. Indian Journal of Radiology and Imaging 2014; 24(3):225-236. 